\title{
Virtual Prototyping of a Compliant Spindle for Robotic Deburring
}

\author{
Giovanni Berselli ${ }^{1, *}$, Marcello Pellicciari ${ }^{2}$, Gabriele Bigi ${ }^{2}$, Angelo O. Andrisano ${ }^{2}$ \\ 1 University of Genova, Via all'Opera Pia 15/A - 16145 Genova, Italy \\ 2 University of Modena and Reggio Emilia, Via Vignolese 905/B, 41125 Modena, Italy \\ * Corresponding E-mail: giovanni.berselli@unige.it
}

\begin{abstract}
At the current state-of-the-art, Robotic Deburring (RD) has been successfully adopted in many industrial applications, but it still needs improvements in terms of final quality. In fact, the effectiveness of a RD process is highly influenced by the limited accuracy of the robot motions and by the unpredictable variety of burr size/shape. Tool compliance partially solves the problem, although dedicated engineering design tools are strictly needed, in order to identify those optimized parameters and RD strategies that allow achieving the best quality and cost-effectiveness. In this context, the present paper proposes a CAD-based Virtual Prototype (VP) of a pneumatic compliant spindle, suitable to assess the process efficiency in different case scenarios. The proposed VP is created by integrating a 3D multibody model of the spindle mechanical structure with the behavioural model of the process forces, as adapted from previous literature. Numerical simulations are provided, concerning the prediction of both cutting forces and surface finishing accuracy.
\end{abstract}

Keywords: CAD-based tools, Compliant Spindle, Robotic Deburring, Virtual Prototyping.

\section{Introduction}

The process of finishing mechanical parts with complex shapes and narrow tolerances generally involves the use of five axes CNC machines, namely extremely expensive devices that require large set-up times. As a potential alternative for the same task, industrial robots offer greater flexibility along with a lower initial investment, but are characterized by an inferior process quality. In the last few years, several efforts have been devoted to the developments of engineering methods and tools for improving the effectiveness of Robotic Deburring (RD) and, more in general, of robotic machining [1].

Focusing on deburring operations, the offline programming of an ideal robot trajectory cannot achieve the best end-product quality, the reasons being the limited motion accuracy of any industrial manipulator and the uneven/unpredictable process condition (i.e.: different burr thickness and varying material properties [2,3]). In practice, a RD process may lead to either partial or excessive deburring (where part of the workpiece is accidently removed). In addition, in case very strict tolerances and good surface roughness are required, a uniform contact pressure between the tool and the workpiece must be guaranteed at all times, despite the burr thickness. In these instances, either an active force feedback [4] or a passive compliant tool are usually adopted, the passive solution being more industrially common thanks to its cost-effectiveness, ease of use and seamless/faster adaptation to unexpected process variations or collisions. Nonetheless, the tuning of the system parameters (e.g. choice of the tool shape, feed-rate, overall compliance) is rather time consuming and requires several physical tests which actually reduce the robotic cell productivity. Therefore, a virtual engineering approach is needed, in order to predict the RD performance without any on-field testing, possibly leading to a "first-time-right", "plugand-produce" technology application. 
For what concerns past literature dealing with deburring processes, a review of several models for the prediction of the cutting forces is reported in [5] (e.g. linear models by Altintas [6] and exponential model by Kienzle [7]), whereas CAD/CAM-assisted methods have been recently proposed in [8]. In any case, most of the previous works simply neglects the influence of the tool compliance, whose complex interaction with the deburring process is experienced only on the physical test rig. Owing to the abovementioned considerations, the present work addresses the development of a CAD-based Virtual Prototype (VP) of a compliant tool coupled with the cutting process, which should allow for the offline optimization of the RD operations. In particular, the behavior of the spindle mechanical structure is modeled by means of a commercial multibody software (Recurdyn), whereas the process forces are concurrently co-simulated in a mathematical simulation environment (Simulink).

\subsection{Description of the Compliant Spindle}

Several commercially available compliant spindles are commonly employed in RD and are usually characterized by pneumatic actuation and radial compliance [3]. The tool considered in this paper, whose detailed description can be found in [9], has been chosen for its widespread adoption and its classical architecture. With reference to the CAD drawing of Fig. 1, the device comprises a pneumatic motor inserted into a housing and supported by a spherical joint (located on point $\mathrm{O}$ ) and by a Compliance Device. The compliance device (Fig. 2) is composed of seven pistons, having limited stroke, and connected to a common chamber with a common air inlet. Initially, as also reported in Sec. 3.3, all the pistons are in direct contact with the pneumatic motor. On the other hand, as depicted in Fig. 3, the pneumatic motor can deflect during interaction with the environment. In this case, some of the pistons may reach their lower dead point, thus loosing contact with the motor (see Fig. 4).

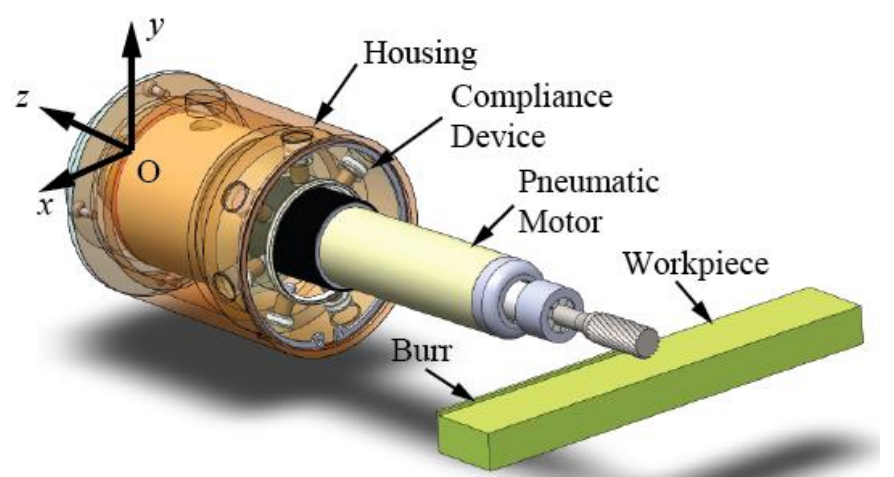

Figure 1. Cad Drawing of the Deburring tool.

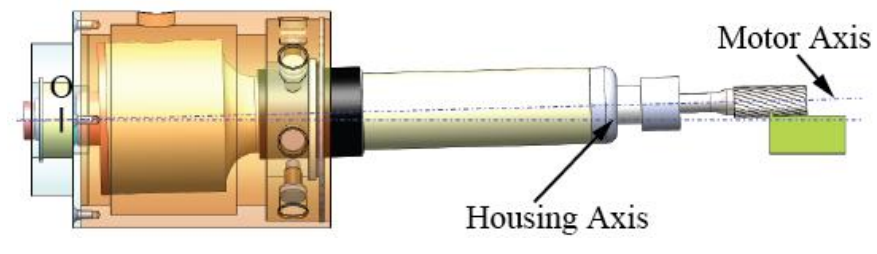

Figure 3. Deburring tool in deflected configuration.

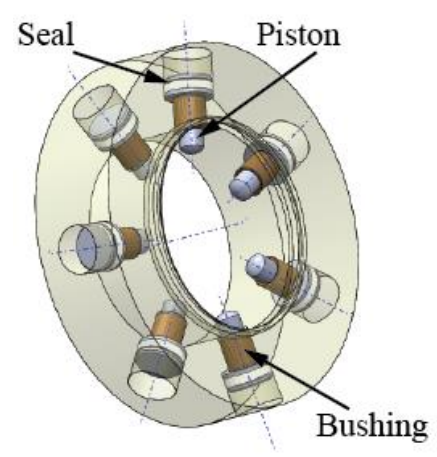

Figure 2. Compliance device.

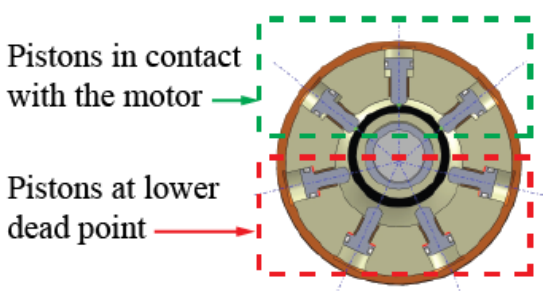

Figure 4. Piston Contacts.

\section{Mechanics of Milling Processes: Background Theory}

Let us first neglect the influence of the system compliance. In this case, the mechanics of milling processes has been extensively treated in [7, pp. 35-46], whose nomenclature is hereafter preserved. With reference to Fig. 5, let us consider an end mill having diameter $D$, helix angle $\beta$ and number of teeth (or flutes) $N$. Let us define $c$ as the process feed rate, $\phi_{j}$ as the instantaneous angle of immersion of the $j$-th tooth within the work piece, $\phi_{p}=2 \pi / N$ as the tooth spacing angle, $\phi_{s t}, \phi_{e x}, \phi_{s}=\left(\phi_{e x}-\phi_{s t}\right)$ as the cutter entry, exit, and swept angles. Although helical mills are usually employed, let us first assume $\beta=0$ and let us consider the $j$-th tooth only. 


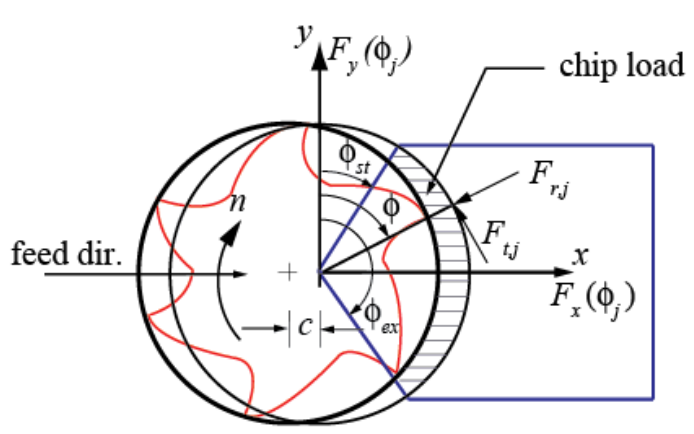

Figure 5. Geometry of milling process [7].

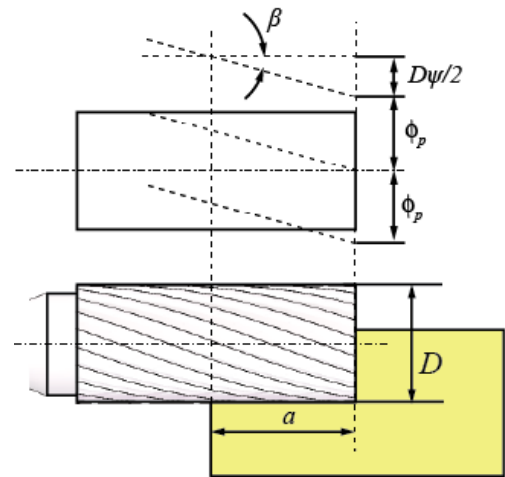

Figure 6. Geometry of helical end milling [7].

In this case, the instantaneous chip thickness, $h_{j}$, can be approximated as $h_{j}\left(\phi_{j}\right)=c \sin \phi_{j}$, whereas tangential, $F_{t, j}$, radial, $F_{r, j}$, and axial, $F_{a, j}$, cutting forces can be expressed as function of edge contact length, $a$, and uncut chip area, $a h\left(\phi_{j}\right)$, such that:

$$
F_{q, j}\left(\phi_{\mathrm{j}}\right)=K_{q, c} a h\left(\phi_{j}\right)+K_{q, e} a, \quad \text { for } q=t, r, a \text { and } j=0, \ldots, N-1
$$

where $K_{t, c}, K_{r, c}$, and $K_{a, c}$ are respectively defined as the cutting force coefficients due to the shearing action in tangential, radial, and axial directions, whereas $K_{t, e}, K_{r, e}$, and $K_{a, e}$ are the edge constants.

Naturally, cutting forces are produced only when the tool is in the cutting (immersion) zone, that is $F_{q, j}\left(\phi_{j}\right)>0$ if $\phi_{s t} \leq \phi_{j} \leq \phi_{e x}$. In addition, multiple teeth will be cutting simultaneously if $\phi_{s}>\phi_{p}$, the overall force being given by the summation of the single $j$-th contribution.

In case an helical mill is used (i.e. $\beta>0$ ), the cutting edge will be lagging behind the tool end point (see Fig. 6). The lag angle, $\psi$, at the axial cut depth, $z$, is $\psi=2 z D^{-1} \tan \beta$. In particular, as stated in [7], when the bottom point of a reference flute is at immersion angle $\phi$, a cutting edge point axially located at a distance $z$ above the reference flute will have an immersion angle of $(\phi-\psi)$. Assuming that the bottom end of one flute is designated as the reference immersion angle $\phi$, the immersion being measured clockwise from the normal $y$ axis, the bottom end points of the remaining flutes are at angles $\phi_{j}(0)=\phi+j \phi_{p}$ for $j=0, \ldots, N-1$. The immersion angle for the $j$-th flute at an axial cut depth $z$ is:

$$
\phi_{j}(z)=\phi+j \phi_{p}-k_{\beta} z \quad \text { where } k_{\beta}=2 D^{-1} \tan \beta
$$

The chip thickness, $h_{j}$, is now approximated as $h_{j}\left(\phi_{j}, z\right)=c \sin \phi_{j}(z)$. Similarly to Eq. 1 , the contribution of the elemental tangential, $d F_{t, j}$, radial, $d F_{r, j}$, and axial, $\mathrm{d} F_{a, j}$, forces on a differential flute element with height $z$ can be written as:

$$
d F_{q, j}\left(\phi_{j}, z\right)=\left[K_{q, c} h_{j}\left(\phi_{j}(z)\right)+K_{q, e}\right] d z, \quad \text { for } q=t, r, a \text { and } j=0, \ldots, N-1
$$

From the equilibrium conditions, the radial and tangential elemental forces can be resolved into feed, $x$, and normal, $y$, directions using the following transformations:

$$
d F_{x, j}=-d F_{t, j} \cos \phi_{j}(z)-d F_{r, j} \sin \phi_{j}(z) ; \quad d F_{y, j}=d F_{t, j} \sin \phi_{j}(z)-d F_{r, j} \cos \phi_{j}(z)
$$

The total force produced by the $j$-th flute can be obtained by integrating the differential cutting forces:

$$
F_{p, j}\left(\phi_{j}(z)\right)=\int_{z_{j, 1}}^{z_{j, 2}} d F_{p, j}\left(\phi_{j}(z)\right) d z, \quad \text { for } p=x, y, z
$$

where $z_{j, 2}\left(\phi_{j}(z)\right)$ and $z_{j, 2}\left(\phi_{j}(z)\right)$ are the lower and upper axial engagement limits of the in-cut portion of the $j$-th flute. The integrations are carried out by noting that $d \phi_{j}(z)=-k_{\beta} z$, so that: 


$$
\begin{aligned}
& F_{x, j}\left(\phi_{j}(z)\right)=\left\{\frac{c}{4 k_{\beta}}\left[-K_{t c} \cos 2 \phi_{j}(z)+K_{r c}\left(2 \phi_{j}(z)-\sin 2 \phi_{j}(z)\right)\right]+\frac{1}{k_{\beta}}\left[K_{t e} \sin \phi_{j}(z)-K_{r e} \cos \phi_{j}(z)\right]\right\}_{z_{j, 1}\left(\phi_{j}(z)\right)}^{z_{j, 2}\left(\phi_{j}(z)\right)} \\
& F_{y, j}\left(\phi_{j}(z)\right)=\left\{\frac{-c}{4 k_{\beta}}\left[-K_{t c}\left(2 \phi_{j}(z)-\sin 2 \phi_{j}(z)\right)+K_{r c} \cos 2 \phi_{j}(z)\right]+\frac{1}{k_{\beta}}\left[K_{t e} \cos \phi_{j}(z)+K_{r e} \sin \phi_{j}(z)\right]\right\}_{z_{j, 1}\left(\phi_{j}(z)\right)}^{z_{j, 2}\left(\phi_{j}(z)\right)} \\
& F_{z, j}\left(\phi_{j}(z)\right)=\frac{1}{k_{\beta}}\left[K_{a c} c \cos \phi_{j}(z)-K_{a e} \phi_{j}(z)\right]_{z_{j, 1}\left(\phi_{j}(z)\right)}^{z_{j,}\left(\phi_{j}(z)\right)}
\end{aligned}
$$

Note that the lag angle at full axial depth of cut (i.e. when $z=a$ ) is $\psi_{a}=k_{\beta} a$. With reference to Fig. 7, the computer algorithm proposed in [7] to determine the axial integration boundaries is as follows:

-If $\phi_{s t}<\phi_{j}(z=0)<\phi_{e x}$, then $z_{j, 1}=0$;

If $\phi_{s t}<\phi_{j}(z=a)<\phi_{e x}$, then $z_{j, 2}=\mathrm{a}$;

If $\phi_{j}(z=a)<\phi_{e x}$, then $z_{j, 2}=\left(1 / k_{\beta}\right)\left(\phi+j \phi_{p}-\phi_{s t}\right)$;

-If $\phi_{j}(z=0)>\phi_{e x}$ and $\phi_{j}(z=a)<\phi_{e x}$ then

$z_{j, 1}=\left(1 / k_{\beta}\right)\left(\phi+j \phi_{p}-\phi_{e x}\right)$;

If $\phi_{j}(z=a)>\phi_{s t}$ then $z_{j, 2}=\mathrm{a}$;

If $\phi_{j}(z=a)<\phi_{s t}$, then $z_{j, 2}=\left(1 / k_{\beta}\right)\left(\phi+j \phi_{p}-\phi_{s t}\right)$;

-If $\phi_{j}(z=0)>\phi_{e x}$ and $\phi_{j}(z=a)>\phi_{e x}$ then the flute is out of cut.

Note that these expressions can be used if flute $j=0$ is aligned at $\phi=0$ in the beginning of the algorithm.

The total instantaneous forces on the cutter at immersion

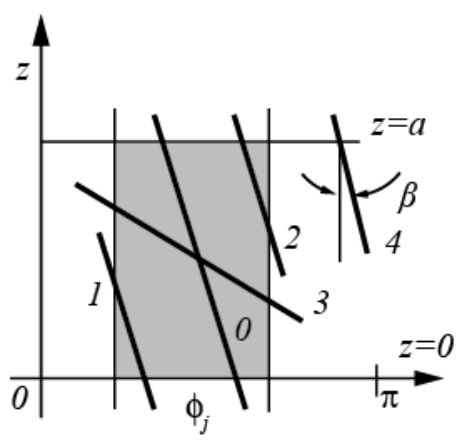

Figure 7. Helical flute-part face integration zones [7]. $\phi$ are finally computed as follows:

$$
F_{x}(\phi)=\sum_{j=0}^{N-1} F_{x_{j}} ; \quad F_{y}(\phi)=\sum_{j=0}^{N-1} F_{y_{j}} ; \quad F_{z}(\phi)=\sum_{j=0}^{N-1} F_{z_{j}} .
$$

\section{Virtual Prototype of the Compliant Spindle}

The spindle VP can be conceptually divided in three subsystems arranged in a loop structure, the output of one subsystem being the input of the following one. With reference to Fig. 8, the model subsystems are used to compute:1) radial burr, $p_{r b}$, and work piece, $p_{r p}$, cut depths; 2) cutting forces; 3 ) cutting tool position and velocity via co-simulation with a CAD-based multi-body software.

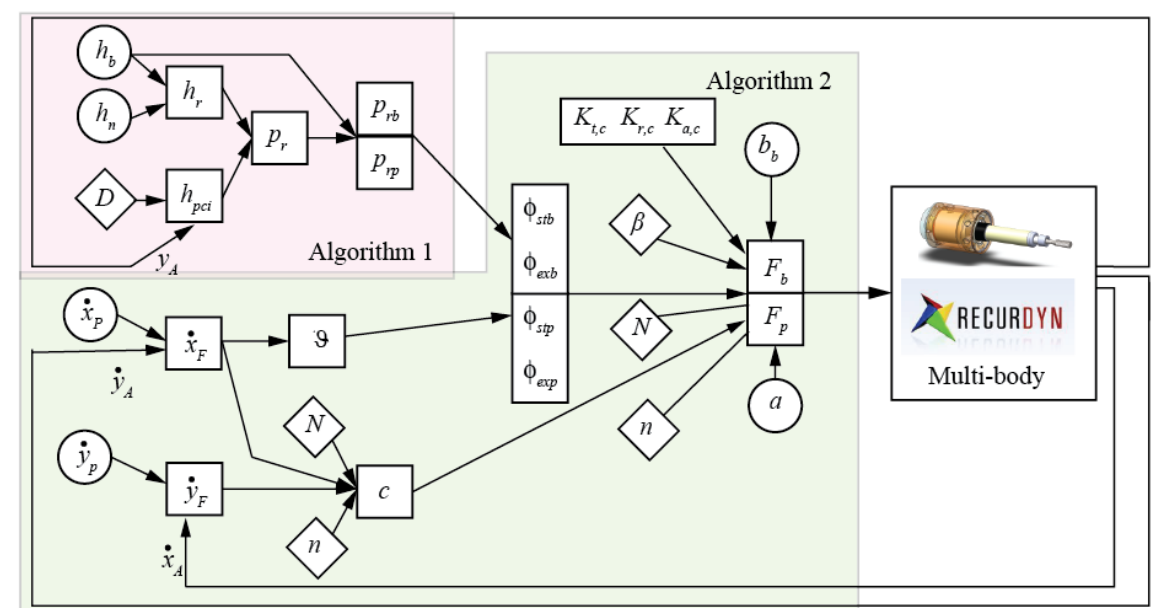

Figure 8. Spindle VP: integration between CAD-based multibody software and process models.

\subsection{Computation of radial burr and workpiece cut depth}

With reference to Fig. 9, let us define a spatial coordinate $w$ and let us suppose to conceptually unroll a $3 \mathrm{D}$ burr profile along that same coordinate. The burr height and width can then be defined as function of 
$w$, that is $h_{b}=h_{b}(w)$ and $b_{b}=b_{b}(w)$, respectively. With reference to Fig. 1 and 10 , let us now define a fixed reference systems, located on point $\mathrm{O}$, the $z$ axis being aligned with the housing symmetry axis, and the $x$ axis indicating the feed direction. Similarly, let us locate the application points of the process forces (as computed in the next algorithm) in A and B. These points lie at the intersections between the mill longitudinal axis (Fig. 10) and the two lines parallel to the $y$ axis and respectively passing through the midpoints of $b_{b}$ and $a$ (the latter being the edge contact length between workpiece and cutter). Note that the position of both points $\mathrm{A}$ and $\mathrm{B}$, with respect to $\mathrm{O}$, can vary due to the spindle compliance. In particular, Fig. 11 depicts a condition where the deburring process is incomplete, whereas Fig. 12 depicts a condition where the mill is cutting both burr and workpiece. With reference to these same figures, let us define, for each time instant, $t$, the variables $x_{A}$ and $y_{A}$ as the $x$ and $y$ coordinates of point $\mathrm{A}$ in the fixed reference systems located on point $\mathrm{O}, h_{p c i}$ as the y coordinate of the mill/workpiece inferior contact point (point C), that defines the workpiece profile after deburring (i.e. $h_{p c i}=y_{A}-D / 2$ ), $h_{r}$ and $h_{n}$ as the real and desired (nominal) workpiece height with respect to O (i.e. $h_{r}=h_{n}-h_{b}$ ), $p_{r}$ as the radial deburring depth $p_{r}=h_{r}-h_{p c i}$. Three cases are possible:

- Ideal deburring: $p_{r}=h_{b}$, so that $p_{r b}=h_{b}$ and $p_{r p}=0$;

- Partial deburring: $p_{r}<h_{b}$, so that $p_{r b}=p_{r}$ and $p_{r p}=0$;

- Excessive deburring: $p_{r}>h_{b}$, so that $p_{r b}=h_{b}$ and $p_{r p}=p_{r}-h_{b}$;

Owing to the abovementioned considerations, the process forces considered hereafter, $\boldsymbol{F}_{b}$ and $\boldsymbol{F}_{p}$, are respectively due to either the mill-burr or the mill-workpiece interaction. The force $\boldsymbol{F}_{b}$, (hereafter named burr force), is applied on point A, whereas the force $\boldsymbol{F}_{p}$, (hereafter named workpiece force), is applied on point $\mathrm{B}$.

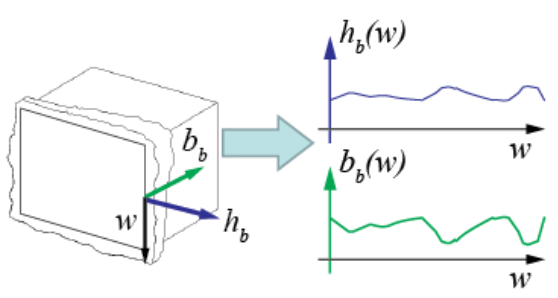

Figure 9. Definition of burr height and width.

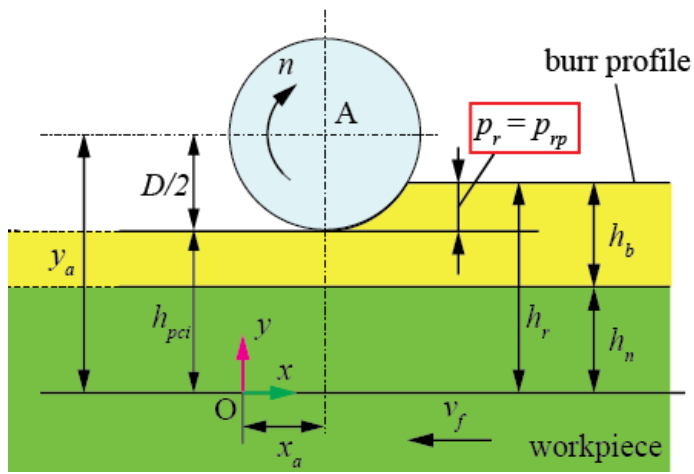

Figure 11. Partial deburring.

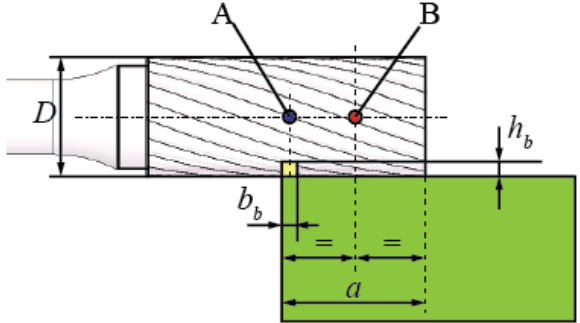

Figure 10. Burr and workpiece geometry.

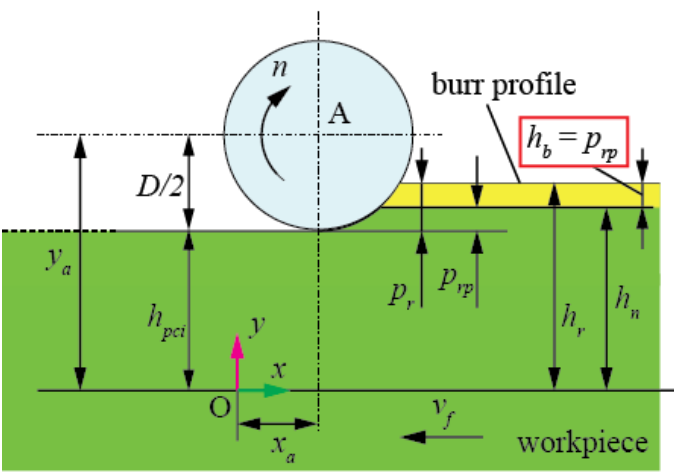

Figure 12. Excessive deburring.

\subsection{Computation of the Deburring Forces}

Due to the presence of a compliant structure, the feed velocity vector will be inclined with respect to the horizontal axis of the workpiece. Let us define $\boldsymbol{v}_{P}=\left[\dot{x}_{P}, \dot{y}_{P}, \dot{z}_{P}\right]^{T}$ and $\boldsymbol{v}_{A}=\left[\dot{x}_{A}, \dot{y}_{A}, \dot{z}_{A}\right]^{T}$ as the relative velocities of workpiece and point $\mathrm{A}$ with respect to the fixed $\mathrm{O}$-frame. The feed velocity vector of the workpiece with respect to the mill is $\boldsymbol{v}_{F}=\boldsymbol{v}_{P}-\boldsymbol{v}_{A}=\left[\dot{x}_{F}, \dot{y}_{F}, \dot{z}_{F}\right]^{T}$. Note that, due to the spindle compliance, $\dot{z}_{F} \neq 0$. Nonetheless, as long as $\dot{z}_{F}$ is always an order of magnitude lower than $\dot{x}_{F}$ and $\dot{y}_{F}$, its contribution is neglected for application purposes (that is $\boldsymbol{v}_{F} \approx\left[\dot{x}_{F}, \dot{y}_{F}, 0\right]^{T}$ ). The angle of inclination, $\vartheta$, of the spindle velocity with respect to the horizontal axis can be evaluated as $\vartheta=\operatorname{atan}\left(\dot{y}_{F} / \dot{x}_{F}\right)$. The process feed rate, $c$, is then given by: 


$$
\begin{aligned}
& c=(N n)^{-1}\left(\dot{x}_{F}{ }^{2}+\dot{y}_{F}{ }^{2}\right)^{1 / 2} \\
& h_{j}\left(\phi_{j}\right)=c \sin \left(\phi_{j}-\vartheta\right)
\end{aligned}
$$

For instance, with reference to Fig. 13, if $\phi_{j}=\phi_{j, 1}=\vartheta$, then $h\left(\phi_{j, 1}, \vartheta\right)=0$. In the same way, if $\phi_{j}=\phi_{j, 2}=\pi / 2+\vartheta$, then $h\left(\phi_{j, 2}, \vartheta\right)=c$. At last, if $\phi_{j}=\phi_{j, 3}=\pi+\vartheta$, then $h\left(\phi_{j, 3}, \vartheta\right)=0$.

Naturally, if the spindle compliance is neglected and, consequently $\dot{y}_{F}=0$, both Eq. 8 and 9 simplifies into the relations given by the standard model from Altintas [7] (recalled in Sec. 2), namely $c=\dot{x}_{F}$ and $h_{j}\left(\phi_{j}\right)=c \sin \phi_{j}$.

The cutting forces can then be found by inserting Eqs. 8-9 into Eq. 3 and performing the necessary calculations for the integration of the elemental forces (Eq. 5). The following expressions are found:

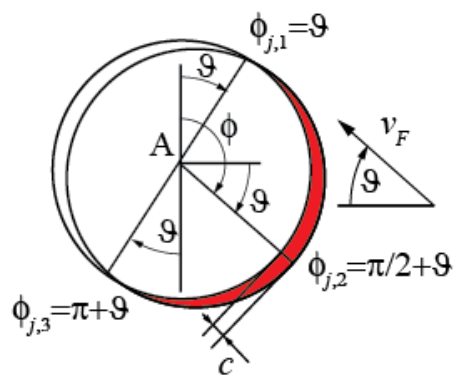

Figure 13. Computation of $h\left(\phi_{j}, \vartheta\right)$

$F_{x, j}\left(\phi_{j}(z)\right)=\left\{\frac{c}{k_{\beta}}\left[K_{t c}\left(-\frac{\phi_{j}(z) \sin (\vartheta)}{2}-\frac{\cos \left(2 \phi_{j}(z)-\vartheta\right)}{4}\right)+K_{r c}\left(\frac{\phi_{j}(z) \cos (\vartheta)}{2}-\frac{\sin \left(2 \phi_{j}(z)-\vartheta\right)}{4}\right)\right]\right\}_{z_{j, 1}\left(\phi_{j}(z)\right)}^{z_{j, 2}\left(\phi_{j}(z)\right)}$

$F_{x, j}\left(\phi_{j}(z)\right)=\left\{\frac{c}{k_{\beta}}\left[K_{t c}\left(-\frac{\phi_{j}(z) \sin (\vartheta)}{2}-\frac{\cos \left(2 \phi_{j}(z)-\vartheta\right)}{4}\right)+K_{r c}\left(\frac{\phi_{j}(z) \cos (\vartheta)}{2}-\frac{\sin \left(2 \phi_{j}(z)-\vartheta\right)}{4}\right)\right]\right\}_{z_{j, 1}\left(\phi_{j}(z)\right)}^{z_{j, 2}\left(\phi_{j}(z)\right)}$

$F_{z, j}\left(\phi_{j}(z)\right)=\frac{1}{k_{\beta}}\left[K_{a c} c \cos \left(\phi_{j}(z)-\vartheta\right)\right]_{z_{j, 1}\left(\phi_{j}(z)\right)}^{z_{j,}\left(\phi_{j}(z)\right)}$

Also in this case, if $\vartheta=0, \mathrm{Eq} 10$ reduces to Eq. 6 . As the last step for the calculation of the cutting forces, the integral limits must be computed. In particular, four integral limits can be defined, $\phi_{s t b}, \phi_{s t p}, \phi_{e x b}, \phi_{\text {exp }}$, which represent the angular immersion of the mill within either the burr or the workpiece.

In case there exists a velocity component in $y$ direction, $\dot{y}_{F}$, the possible instantaneous cases are depicted in Tab. 1, according to the value of $\vartheta$ (either zero, positive or negative) or to the values of $p_{r p}$ and $p_{r b}$.

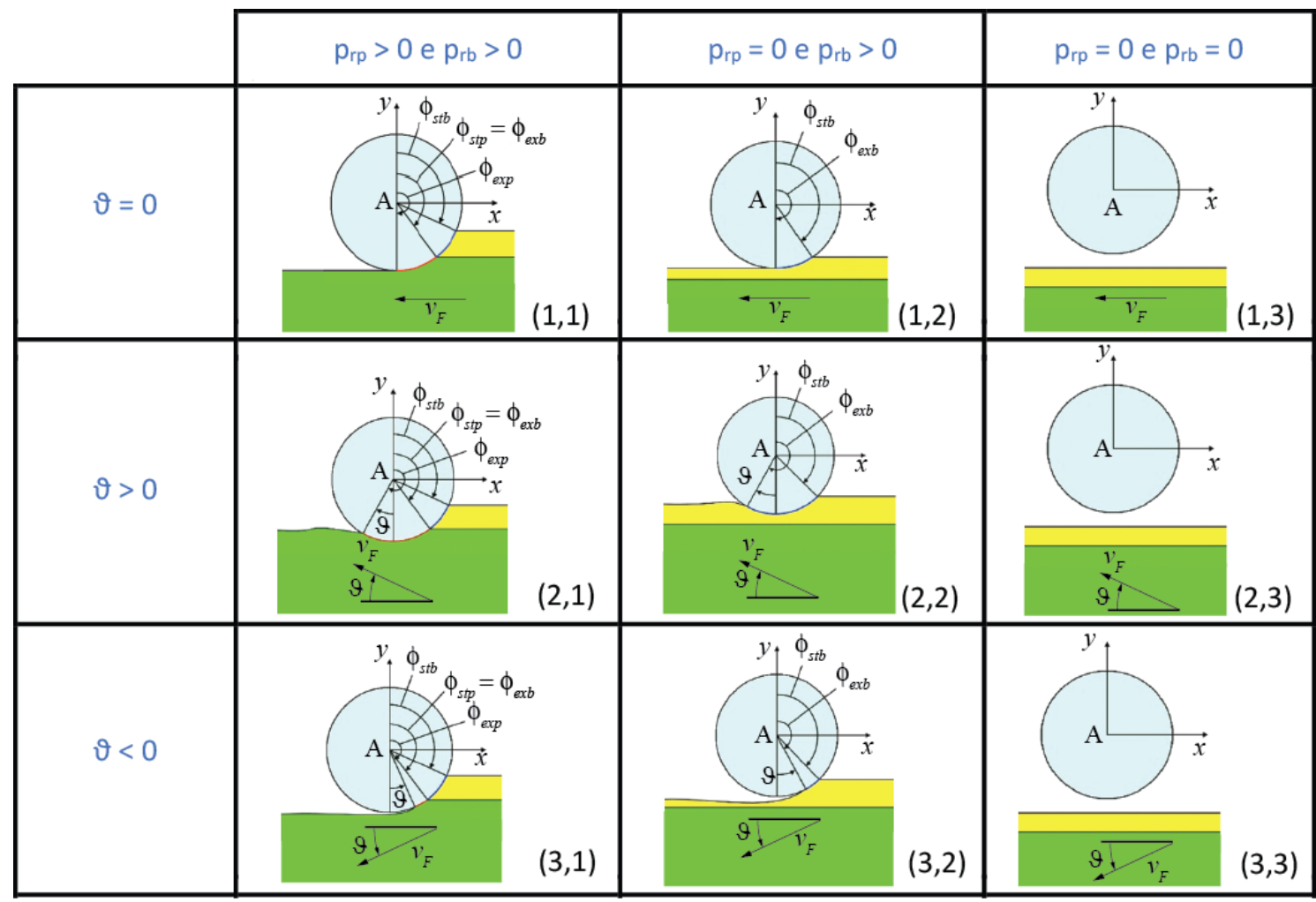

Table 1. Possible cases for the deburring process 
For what concerns the cases depicted in the third column, it is not necessary to compute any integral limit, the mill not being in contact with either burr or workpiece (i.e. $\boldsymbol{F}_{b}=\boldsymbol{F}_{p}=0$.) In parallel, the cases portrayed in the second column depict a situation where the mill is in contact with the burr only, so that $\phi_{s t p}$ and $\phi_{\text {exp }}$ are not defined (i.e. $\boldsymbol{F}_{p}=0$ ). In summary, the overall algorithm for the computation of the integral limits, which requires $, p_{r p}, p_{r b}$, and $h_{b}$ as inputs, is formulated as follows:

$$
\begin{array}{ll}
\varphi_{s t b}=\pi-\operatorname{acos}\left(1-\frac{2 p_{r}}{D}\right), \text { always } ; & \varphi_{\text {exb }}=\left\{\begin{aligned}
\pi-\operatorname{acos}\left(1-\frac{2 p_{r p}}{D}\right), & \text { if } p_{r}>h_{b} \\
\pi+\vartheta, & \text { if } 0<p_{r}<h_{b}
\end{aligned}\right. \\
\varphi_{\text {stp }}=\pi-\operatorname{acos}\left(1-\frac{2 p_{r p}}{D}\right), \text { if } p_{r}>h_{b} ; \quad \varphi_{\text {exp }}=\pi+\vartheta, \text { if } p_{r}>h_{b}
\end{array}
$$

\subsection{CAD-based multibody model}

As depicted in Fig. 8, the CAD-based multi-body model of the spindle mechanical structure computes mill position and velocity (specifically of point $\mathrm{A}$ and $\mathrm{B}$ ) for given workpiece, $\boldsymbol{F}_{p}$, and burr, $\boldsymbol{F}_{b}$, forces. The multi-body model describes the kinematic structure of the spindle, the dynamics of every moving body, and the internal forces due to frictions, contacts, and internal pressure in the chamber of the compliant device. As for the spindle kinematic structure, the housing is considered as fixed (connected to the ground), the pneumatic motor is connected to the housing via a spherical joint on point $\mathrm{O}$, the mill rotates with a given velocity $n$. The seven pistons can translate along their axis. Three possible contacts are imposed to each piston, namely contact with the pneumatic motor (point $\mathrm{C}$ ), and possible contacts with the chamber at either the lower or the upper dead-points, see Fig. 2, 4 and 16. At the initial (undeflected) spindle configuration, all the pistons are in contact with the motor.

Concerning the internal forces, two forces have been included:

- Pressure on the piston dome, $F_{p s t}$, simply given as $F_{p s t}=A_{p s t} p$, the parameter $A_{p s t}$ and $p$ being piston dome area and chamber pressure.

- Friction force on the piston rubber seals, $F_{s l n}$. Note that, as depicted in Fig. 14, the seals present an hollow structure subjected to a pressure $p$. The force, $F_{s l n}^{\prime}$, having direction perpendicular to that of the piston motion and due to the chamber-seal interaction, is computed as $F_{s l n}^{\prime}=A_{s l n} p+\mathrm{P}$, the parameter $A_{s l n}$ and $P$ being the seal lateral area and preload. The force, $F_{s l n}$, having direction parallel to that of the piston motion and due to friction, is given by $F_{s l n}=\mu F_{s l n}^{\prime}$, the parameter $\mu$ being either the static or dynamic coefficient of friction.
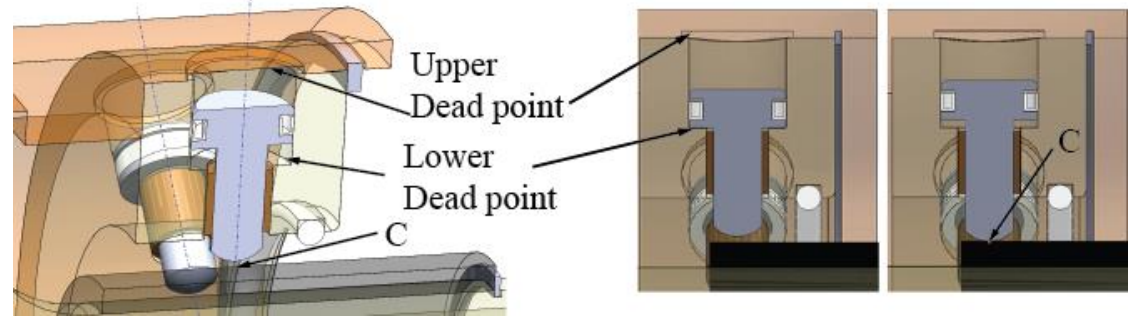

Figure 14. Contact points

\section{Numerical Simulations}

At this stage, the VP is validated via a set of numerical simulations. The following parameters have been used: $K_{t, c}=2000 \mathrm{~N} / \mathrm{mm}^{2}, K_{r, c}=1200 \mathrm{~N} / \mathrm{mm}^{2}, K_{a, c}=800 \mathrm{~N} / \mathrm{mm}^{2}, K_{t, e}=K_{r, e}=K_{a, e}=0, N=20, n=40.000 \mathrm{rpm}$, $D=8 \mathrm{~mm}, a=10 \mathrm{~mm}, h_{b}=b_{b}=1 \mathrm{~mm}, h_{n}=5 \mathrm{~mm}, v_{f}=80 \mathrm{~mm} / \mathrm{s}, p=5$ bar, $\beta=20^{\circ}, F_{p s t}=7.70 \mathrm{~N}, F_{s l n}=3.90 \mathrm{~N}$ (static friction) or $F_{s l n}=2.80 \mathrm{~N}$ (dynamic friction). Let us define the process error as

$$
e=\min \left(h_{p c i}-h_{n}, h_{b}\right)
$$


A positive error indicates a partial deburring, whereas a negative error indicates an excessive deburring. As an example, Fig. 15 depicts a graph of the burr force components $F_{b, x}, F_{b, y}, F_{b, z}$, which underlines how the process forces stabilize after an initial transient. Figure 16 depicts the process error, which stabilizes on a sufficiently low negative value.

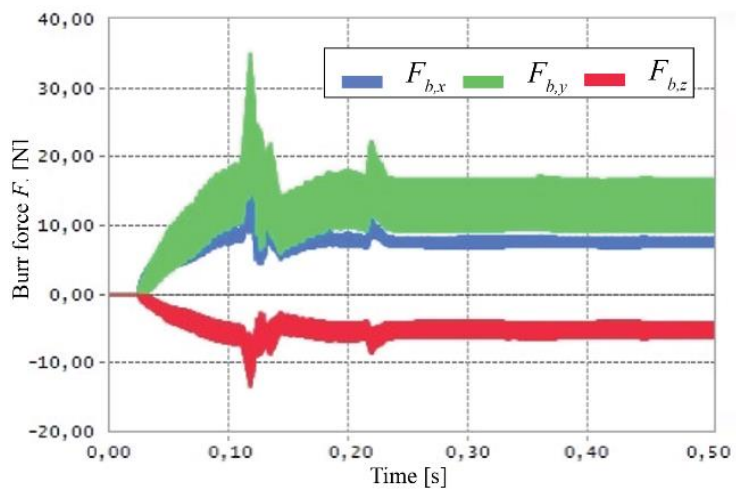

Figure 15. Burr force components.

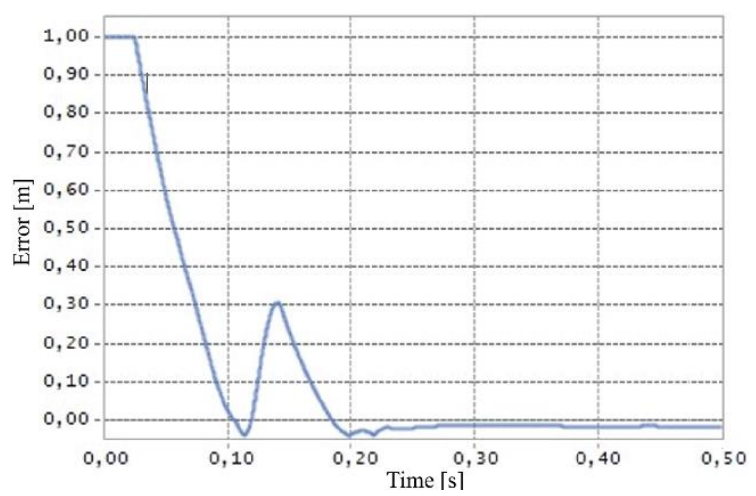

Figure 16. Surface finishing error.

\section{Conclusions}

A CAD-based Virtual Prototype (VP) of a pneumatic compliant spindle has been presented, which is based on a co-simulation model employing a commercial multibody software along with a mathematical simulation environment. The VP can effectively predict both deburring forces and finishing errors, thus enabling for a virtual test of the process quality. In addition, the versatility of the CAD environment allows to easily evaluate the influence of several design (and control) parameters, such as the overall spindle compliance and the influence of friction in the sliding pairs. Future research will be devoted to the experimental validation of the proposed VP.

\section{Ackowledgments}

This research was funded by the Italian Ministry of research within the project "Adaptive and modular approaches for the digital enabled factory", CTN01_00163_216730.

\section{References}

[1] J. Pandremenos, C. Doukas, P. Stavropoulos, G. Chryssolouris, "Machining with Robots: A Critical Review, Proc. of DET2011, 7th Int. Conf. on Digital Enterprise Tech., pp. 1-8, Sept. 28-30, 2011.

[2] M. Mohammad, V. Babriya, T. Sobh, "Modeling A Deburring Process, Using DELMIA V5," Technological Developments in Education and Automation, pp 549-558, 2010.

[3] B. Ryuh and G. R. Pennock, "Robot Automation Systems for Deburring," Industrial Robotics: Programming, Simulation and Applications, L. K. Huat (Ed.), ISBN: 3-86611-286-6, InTech, 2006.

[4] L. Liang, F. Xi, K. Liu, "Modeling and Control of Automated Polishing/deburring Process Using a Dual-Purpose Compliant Toolhead," International Journal of Machine Tools and Manufacture, vol. 48, no. $12-13,2008$.

[5] B. Denkena e F. Hollmann, Process Machine Interactions - Predicition and Manipulation of Interactions between Manufacturing Processes and Machine Tool Structures, Springer, 2013.

[6] Y. Altintas, Manufacturing automation, Metal Cutting Mechanics, Machine Tool Vibrations, and CNC Design, New York: Cambridge University Press, 2012.

[7] Kienzle, O. and Victor, H., "Spezifische Shnittkraefte bei der Metall-bearbeitung", Werkstattstehnik und Maschinenbau, Bd. 47, H.5, 1957.

[8] K. Schützer, E Abele, S Güth, "Simulation-based deburring tool and process development," CIRP Annals - Manufacturing Technology, vol. 64, no. 1, pp. 357-360, 2015.

[9] D. K. Lawson, "Deburring tool," U.S. Patent 6,974,286 B2, filed Jul. 25, 2003, and issued Dec. 13, 2005. 